\title{
Results for Two-Level Designs with General Minimum Lower-Order Confounding
}

\author{
Zhi Ming Li ${ }^{1}$ and Run Chu Zhang ${ }^{2,3}$ \\ ${ }^{1}$ School of Mathematical Sciences, Xinjiang University, Urumqi 830046, China \\ ${ }^{2}$ KLAS and School of Mathematics, Northeast Normal University, Changchun 130024, China \\ ${ }^{3}$ LPMC and School of Mathematical Sciences, Nankai University, Tianjin 300071, China \\ Correspondence should be addressed to Zhi Ming Li; zhimingli525@sina.com
}

Received 2 June 2014; Accepted 19 November 2014

Academic Editor: Haijun Jiang

Copyright (C) 2015 Z. M. Li and R. C. Zhang. This is an open access article distributed under the Creative Commons Attribution License, which permits unrestricted use, distribution, and reproduction in any medium, provided the original work is properly cited.

\begin{abstract}
The general minimum lower-order confounding (GMC) criterion for two-level design not only reveals the confounding information of factor effects but also provides a good way to select the optimal design, which was proposed by Zhang et al. (2008). The criterion is based on the aliased effect-number pattern (AENP). Therefore, it is very important to study properties of AENP for two-level GMC design. According to the ordering of elements in the AENP, the confounding information between lower-order factor effects is more important than that of higher-order effects. For two-level GMC design, this paper mainly shows the interior principles to calculate the leading elements ${ }_{1}^{\#} C_{2}$ and ${ }_{2}^{\#} C_{2}$ in the AENP. Further, their mathematical formulations are obtained for every GMC $2^{n-m}$ design with $N=2^{n-m}$ according to two cases: (i) $5 N / 16+1 \leq n<N / 2$ and (ii) $N / 2 \leq n \leq N-1$.
\end{abstract}

\section{Introduction}

To find optimal designs in a more elaborate and explicit manner under effect hierarchy principle, Zhang et al. [1] first introduced the aliased effect-number pattern (AENP) and proposed a new criterion of general minimum lower-order confounding (GMC) for two-level regular design. Further, they proved that all the classification patterns conducting the existing criteria, such as maximum resolution (MR) criterion [2], minimum aberration (MA) criterion [3], clear effects (CE) criterion [4], and maximum estimation capacity (MEC) criterion [5], can be expressed as different functions of the AENP so that it can be a basis to unify these criteria.

Through the AENP, we can get a deeper understanding of properties of the above criteria and relationships among them. Zhang and Cheng [6] revealed an exact expression of the average minimum lower-order confounding property of MA design. Hu and Zhang [7] obtained an essential statistical equivalence of MEC design and MA design. From the average least confounding property between lower-order effects, MA designs are most suitable for the situation that all the factors in experiments are treated to be equally important, while GMC design has an individual least confounding property between lower-order effects and possesses the maximum numbers of clear main effects and clear two-factor interactions (2fi's). Because of this, GMC designs can be applied to the experiments which the experimenters have some prior information to the order of the importance factors. In practice, the latter situation more often happens than the former one. Therefore, the study for GMC designs should be significantly important in both theory and application.

Now we review some definitions proposed by Zhang et al. [1]. Let $D$ be a $2^{n-m}$ design with $n$ factors, $m$ independent defining words, and $N=2^{n-m}$ runs. We denote the factors by $1,2, \ldots, n$. An $i$ th-order factor effect is said to be aliased with $j$ th-order factor effects at degree $k$ if it is simultaneously aliased with $k j$ th-order factor effects. The 0 th-order effect is the grand mean and 1st-order effect is a main effect.

Let ${ }_{i}^{\#} C_{j}^{(k)}(D)$ (written by ${ }_{i}^{\#} C_{j}^{(k)}$ for short) be the number of $i$ th-order factor effects that are aliased with $k j$ th-order factor effects. Denote $K_{j}=\left(\begin{array}{c}n \\ j\end{array}\right)$; a set $\left\{{ }_{i}^{\#} C_{j}^{(k)}, 0 \leq k \leq K_{j}\right.$, 
$0 \leq i, j \leq n\}$ is called the aliased effect-number pattern (AENP) of the design $D$. The set reflects the overall confounding between factor effects in the design. Define ${ }_{i}^{\#} C_{j}=$ $\left({ }_{i}^{\#} C_{j}^{(0)},{ }_{i}^{\#} C_{j}^{(1)}, \ldots,{ }_{i}^{\#} C_{j}^{\left(K_{j}\right)}\right)$ and a design that sequentially maximizes the vector

$$
{ }^{\#} \mathrm{C}=\left({ }_{1}^{\#} C_{2},{ }_{2}^{\#} C_{2},{ }_{1}^{\#} C_{3},{ }_{2}^{\#} C_{3},{ }_{3}^{\#} C_{2},{ }_{3}^{\#} C_{3}, \ldots\right)
$$

is called a GMC design, where the ordering of ${ }_{i}^{\#} C_{j}$ 's is in accordance with the rule: ${ }_{i}^{\#} C_{j}$ is before ${ }_{u}^{\#} C_{v}$ if either $\max (i, j)<$ $\max (u, v)$, or $\max (i, j)=\max (u, v)$, with $i<u$, or $\max (i, j)=$ $\max (u, v)$ with $i=u$ and $j<v$. In order to make main effects or 2fi's estimable, we need to give an assumption: the interactions involving three or more factors are absent. Thus, we only study the leading terms ${ }_{1}^{\#} C_{2}$ and ${ }_{2}^{\#} C_{2}$ of AENP for two-level GMC design in this paper.

Zhang et al. [1] listed all two-level GMC designs of 16 and 32 runs, a number of 64-run GMC designs, and obtained the values of ${ }_{1}^{\#} C_{2}$ and ${ }_{2}^{\#} C_{2}$ by computer algorithm. However, the method is not suitable for designs with larger runs. Zhang and Cheng [6] and Chen and Liu [8] provided an important theory for constructing GMC designs. Cheng and Zhang [9] and Li et al. [10] finished the construction of GMC $2^{n-m}$ designs with $N / 4+1 \leq n \leq N-1$. However, there are few articles that pay attention to calculating the values of elements in the AENP, especially, the confounding information between main effects and 2fi's, or among 2fi's of two-level GMC design.

This paper mainly reveals the interior principles for calculating the values of ${ }_{1}^{\#} C_{2}$ and ${ }_{2}^{\#} C_{2}$ for two-level GMC design. In Section 2, we introduce some notations and obtain useful lemmas to study the lower-order confounding information of two-level GMC designs. Section 3 and Section 4, respectively, obtain values of ${ }_{1}^{\#} C_{2}$ and ${ }_{2}^{\#} C_{2}$ for GMC $2^{n-m}$ design with resolution $R \geq I I I$, for $5 N / 16+1 \leq n<N / 2$ and $N / 2 \leq$ $n \leq N-1$. Concluding remarks are given in Section 5 .

\section{Some Notations and Lemmas}

Denote $q=n-m$ and $1,2, \ldots, q$ stand for $q$ independent factors. Let $H_{q}$ be the set containing all main effects $1,2, \ldots, q$ and all interactions among them, formed by

$$
H_{1}=\{1\}, \quad H_{q}=\left\{H_{q-1}, q, q H_{q-1}\right\},
$$

where $q H_{q-1}=\left\{q d: d \in H_{q-1}\right\}$. By Theorem 2.7.1 of Mukerjee and $\mathrm{Wu}$ [11], any $2^{n-m}$ design $D$ can be represented by an $n$ subset of $H_{q}$; that is, $D \subset H_{q}$.

Let $T_{1}=H_{1}$ and $T_{r}=\left\{r, r H_{r-1}\right\}$ for $1<r \leq q$. Evidently, $H_{q}=\cup_{r=1}^{q} T_{r}$. For $5 N / 16+1 \leq n \leq N-1$, Li et al. [10] have gotten that every GMC $2^{n-m}$ design is constructed by the last $n$ columns of $H_{q}$. Therefore, GMC $2^{n-m}$ designs with $5 N / 16+1 \leq n<N / 2$ are directly formed by the last $n$ columns of $T_{q}$. Denote $S_{q r}=H_{q} \backslash H_{r}$ with $1 \leq r<q$. For $N / 2 \leq n \leq N-1$, there exists a number $r(<q)$ so that GMC $2^{n-m}$ design is formed by the last $n$ columns of $T_{r} \cup S_{q r}$. Thus, the GMC design can be written by $D_{0} \cup S_{q r}$, where $D_{0}$ consists of the last $n-\left(N-2^{r}\right)$ columns of $T_{r}$. To get the lower-order confounding information of two-level GMC design, we need to study structure of last $n_{0}$ columns of $T_{r}$ for $r \leq q$ and $n_{0} \leq n$.

Suppose $D_{0}$ consists of the last $n_{0}$ columns of $T_{r}(r \leq q)$, where $n_{0}=\#\left\{D_{0}\right\}$ and $\# A$ denotes the cardinality of a set $A$. The following example illustrates the structure of $D_{0}$.

Example 1. Consider $r=7$; we select the last $n_{0}$ columns of $T_{7}$ to construct $D_{0}$. Clearly, there are 64 choices besides $D_{0} \equiv T_{r}$. For $1 \leq n_{0} \leq 63, D_{0}$ is one of the following six forms.

(i) $(u+1) \cdots 7 H_{u}$ for $1 \leq u<7$.

(ii) $(u+1) \cdots 7\left(H_{u} \backslash H_{v}\right)$ for $1 \leq v<u<7$.

(iii) $(u+1) \cdots 7\left((s+1) \cdots v H_{s} \cup\left(H_{u} \backslash H_{v}\right)\right)$ for $1 \leq s<v<$ $u<7$.

(iv) $(u+1) \cdots 7\left((s+1) \cdots v\left(H_{s} \backslash H_{t}\right) \cup\left(H_{u} \backslash H_{v}\right)\right)$ for $1 \leq$ $t<s<v<u<7$.

(v) $(u+1) \cdots 7\left((s+1) \cdots v\left((w+1) \cdots t H_{w} \cup\left(H_{s} \backslash H_{t}\right)\right) \cup\right.$ $\left.\left(H_{u} \backslash H_{v}\right)\right)$ for $1 \leq w<t<s<v<u<7$.

(vi) $(u+1) \cdots 7\left((s+1) \cdots v\left((w+1) \cdots t\left(H_{w} \backslash H_{z}\right) \cup\left(H_{s} \backslash\right.\right.\right.$ $\left.\left.\left.H_{t}\right)\right) \cup\left(H_{u} \backslash H_{v}\right)\right)$ for $1 \leq z<w<t<s<v<u<7$.

The above example provides a way to construct $D_{0}$. Generally, for any $r(r \leq q)$, we consider the construction of $D_{0}$ in $T_{r}$. Define

$$
\begin{array}{r}
D_{t}=H_{i_{t}} \backslash H_{j_{t}}, \quad a_{i_{t}}=\left(i_{t}+1\right)\left(i_{t}+2\right) \\
\cdots j_{t-1}, \\
1 \leq t \leq m,
\end{array}
$$

where $1 \leq j_{m}<i_{m}<j_{m-1}<i_{m-1}<\cdots<j_{t}<i_{t}<\cdots<$ $j_{1}<i_{1}<j_{0}=r$. Then, $D_{0}$ can be constructed by either of the following cases.

Case 1. One has $D_{0}=a_{i_{1}}\left(a_{i_{2}}\left(\cdots\left(a_{i_{m-1}}\left(a_{i_{m}} D_{m} \cup D_{m-1}\right) \cdots\right) \cup\right.\right.$ $\left.\left.D_{2}\right) \cup D_{1}\right)$.

Case 2. One has $D_{0}=a_{i_{1}}\left(a_{i_{2}}\left(\cdots\left(a_{i_{m-1}}\left(a_{i_{m}} H_{i_{m}} \cup D_{m-1}\right) \cdots\right) \cup\right.\right.$ $\left.\left.D_{2}\right) \cup D_{1}\right)$.

In Case 1 , the number of elements in $D_{0}$ is even since $\#\left\{D_{0}\right\}=\sum_{t=1}^{m}\left(2^{i_{k}}-2^{j_{k}}\right)$. However, that of $D_{0}$ in Case 2 is odd because of $\#\left\{D_{0}\right\}=\sum_{t=1}^{m-1}\left(2^{i_{k}}-2^{j_{k}}\right)+2^{i_{m}}-1$.

Consider $D \subset H_{q}$ and any $\gamma \in H_{q}$; define

$$
B_{2}(D, \gamma)=\#\left\{\left(d_{1}, d_{2}\right): d_{1}, d_{2} \in D, d_{1} d_{2}=\gamma\right\} \text {, }
$$

which is the number of 2fi's in $D$ aliased with $\gamma$. By the definition of ${ }_{i}^{\#} C_{j}^{(k)}(D)$, it can be easily obtained that

$$
\begin{gathered}
{ }_{1}^{\#} C_{2}^{(k)}(D)=\#\left\{\gamma: \gamma \in D, B_{2}(D, \gamma)=k\right\}, \\
{ }_{2}^{\#} C_{2}^{(k)}(D)=(k+1) \#\left\{\gamma: \gamma \in H_{q}, B_{2}(D, \gamma)=k+1\right\},
\end{gathered}
$$

where $k=0,1, \ldots, K_{2}$. In order to get the lower-order confounding of $D_{0}$ in the above cases, we need to study $B_{2}\left(D_{t}, \gamma\right)$ for $t \geq 1$. 
Lemma 2. Let $D_{t}$ be defined in (3) for $t \geq 1$. Then

$$
B_{2}\left(D_{t}, \gamma\right)= \begin{cases}2^{i_{t}-1}-2^{j_{t}-1}, & \gamma \in H_{j_{t}}, \\ 2^{i_{t}-1}-2^{j_{t}}, & \gamma \in H_{i_{t}} \backslash H_{j_{t}}, \\ 0, & \gamma \in H_{q} \backslash H_{i_{t}} .\end{cases}
$$

Proof. For $\gamma \in H_{q} \backslash H_{i_{t}}$, we have $B_{2}\left(D_{t}, \gamma\right)=0$. If $\gamma \in H_{j_{t}}$, then

$$
B_{2}(D, \gamma)=\frac{\#\left\{H_{i_{t}} \backslash H_{j_{t}}\right\}}{2}=2^{i_{t}-1}-2^{j_{t}-1}
$$

For $\gamma \in H_{i_{t}} \backslash H_{j_{t}}$, there are $2^{i_{t}-1}-1$ pairs of factors in $H_{i_{t}}$ so that their interactions are aliased with $\gamma$. Among these pairs, there are $2^{j_{t}}-1$ pairs with one factor from $H_{j_{t}}$ and another from $H_{i_{t}} \backslash H_{j_{t}}$. Thus,

$$
B_{2}\left(D_{t}, \gamma\right)=\left(2^{i_{t}-1}-1\right)-\left(2^{j_{t}}-1\right)=2^{i_{t}-1}-2^{j_{t}} .
$$

This completes the proof. denote

Next we analyze Case 1 of $D_{0}$. For convenience, by (3),

$$
\mathscr{D}(t)=a_{i_{t}}\left(\cdots\left(a_{i_{m-1}}\left(a_{i_{m}} D_{m} \cup D_{m-1}\right) \cdots\right) \cup D_{t}\right)
$$

for $1<t \leq m$. Evidently, $\mathscr{D}(t) \subset H_{j_{t-1}}$ and $\mathscr{D}(1)=D_{0}$ in Case 1. When $d_{1} \in \mathscr{D}(t)$ and $d_{2} \in D_{t-1}$, we have $d_{1} d_{2} \in D_{t-1}$. Thus,

$$
\#\left\{\left(d_{1}, d_{2}\right): d_{1} \in \mathscr{D}(t), d_{2} \in D_{t-1}, d_{1} d_{2}=\gamma\right\}=\sum_{l=t}^{m} \#\left\{D_{l}\right\}
$$

for $\gamma \in D_{t-1}$. Otherwise, the value is zero. Then

$$
\begin{gathered}
\#\left\{\left(d_{1}, d_{2}\right): d_{1} \in \mathscr{D}(t), d_{2} \in D_{t-1}, d_{1} d_{2}=\gamma\right\} \\
= \begin{cases}\sum_{l=t}^{m}\left(2^{i_{l}}-2^{j_{l}}\right), & \gamma \in D_{t-1}, \\
0, & \gamma \notin D_{t-1} .\end{cases}
\end{gathered}
$$

Based on Lemma 2 and (12), we can get the following result for Case 1.

Lemma 3. Let $D_{0}=a_{i_{1}}\left(a_{i_{2}}\left(\cdots\left(a_{i_{m-1}}\left(a_{i_{m}} D_{m} \cup D_{m-1}\right) \cdots\right) \cup\right.\right.$ $\left.\left.D_{2}\right) \cup D_{1}\right)$. Then

$$
\begin{aligned}
& B_{2}\left(D_{0}, \gamma\right) \\
& = \begin{cases}\frac{c(m+1)}{2}, & \gamma \in H_{j_{m}}, \\
c(m+1)-\frac{\left(c(t)+2^{i_{t}}\right)}{2}, & \gamma \in D_{t}, t=1, \ldots, m, \\
\frac{c(t)}{2}, & \gamma \in H_{j_{t-1}} \backslash H_{i_{t}}, t=2, \ldots, m, \\
0, & \gamma \in H_{q} \backslash H_{i_{1}},\end{cases}
\end{aligned}
$$

where

$$
c(1)=0, \quad c(t)=\sum_{l=1}^{t-1}\left(2^{i_{l}}-2^{j_{l}}\right), \quad t>1
$$

Proof. For $1<l \leq m$, by (10), we have

$$
\begin{aligned}
B_{2}\left(a_{i_{l}}\left(\mathscr{D}(l) \cup D_{l-1}\right), \gamma\right) \\
=B_{2}\left(\mathscr{D}(l) \cup D_{l-1}, \gamma\right) \\
=B_{2}(\mathscr{D}(l), \gamma)+B_{2}\left(D_{l-1}, \gamma\right) \\
\quad+\#\left\{\left(d_{1}, d_{2}\right): d_{1} \in \mathscr{D}(l), d_{2} \in D_{l-1}, d_{1} d_{2}=\gamma\right\} .
\end{aligned}
$$

Hence,

$$
\begin{aligned}
B_{2}( & \left.D_{0}, \gamma\right) \\
= & \sum_{l=1}^{m-2} B_{2}\left(D_{l}, \gamma\right)+B_{2}\left(a_{i_{m}} D_{m} \cup D_{m-1}, \gamma\right) \\
& +\sum_{l=2}^{m-1} \#\left\{\left(d_{1}, d_{2}\right): d_{1} \in \mathscr{D}(l), d_{2} \in D_{l-1}, d_{1} d_{2}=\gamma\right\} \\
= & \sum_{l=1}^{m} B_{2}\left(D_{l}, \gamma\right) \\
& +\sum_{l=2}^{m} \#\left\{\left(d_{1}, d_{2}\right): d_{1} \in \mathscr{D}(l), d_{2} \in D_{l-1}, d_{1} d_{2}=\gamma\right\} .
\end{aligned}
$$

Put $H_{r}$ into $2 m+1$ incompatible parts: $H_{j_{m}}, D_{l+1}$, and $H_{j_{l}} \backslash H_{i_{l+1}}$ for $l=0,1, \ldots, m-1$. Clearly, if $\gamma \in H_{q} \backslash H_{i_{1}}$, then $B_{2}\left(D_{0}, \gamma\right)=0$. By Lemma 2 and (12), we, respectively, discuss the following cases.

(i) If $\gamma \in H_{j_{m}}$, then

$$
\#\left\{\left(d_{1}, d_{2}\right): d_{1} \in \mathscr{D}(l), d_{2} \in D_{l-1}, d_{1} d_{2}=\gamma\right\}=0
$$

for $1<l \leq m-1$. Thus,

$$
B_{2}\left(D_{0}, \gamma\right)=\sum_{l=1}^{m} B_{2}\left(D_{t}, \gamma\right)=\sum_{l=1}^{m}\left(2^{i_{l}-1}-2^{j_{l}-1}\right) .
$$

(ii) If $\gamma \in D_{t}$ with $1<t \leq m$, one has

$$
\begin{aligned}
B_{2}( & \left.D_{0}, \gamma\right) \\
= & \sum_{l=1}^{t-1} B_{2}\left(D_{l}, \gamma\right)+B_{2}\left(D_{t}, \gamma\right) \\
& +\#\left\{\left(d_{1}, d_{2}\right): d_{1} \in \mathscr{D}(t+1), d_{2} \in D_{t}, d_{1} d_{2}=\gamma\right\} \\
= & \sum_{l=1}^{t-1}\left(2^{i_{l}-1}-2^{j_{l}-1}\right)+\left(2^{i_{t}-1}-2^{j_{t}}\right) \\
& +\sum_{l=t+1}^{m}\left(2^{i_{l}}-2^{j_{l}}\right) \\
= & \sum_{l=1}^{m}\left(2^{i_{l}}-2^{j_{l}}\right)-\sum_{l=1}^{t-1}\left(2^{i_{l}-1}-2^{j_{l}-1}\right)-2^{i_{t}-1} .
\end{aligned}
$$


(iii) If $\gamma \in H_{j_{t-1}} \backslash H_{i_{t}}$ for $t>1$, then

$$
B_{2}\left(D_{0}, \gamma\right)=\sum_{l=1}^{t-1} B_{2}\left(D_{l}, \gamma\right)=\sum_{l=1}^{t-1}\left(2^{i_{l}-1}-2^{j_{l}-1}\right) \text {. }
$$

This completes the proof.

Lemma 3 shows that the value of $B_{2}\left(D_{0}, \gamma\right)$ in Case 1 depends on all pairs $\left\{i_{t}, j_{t}\right\}_{1 \leq t \leq m}$ which relate to $\#\left\{D_{0}\right\}=\sum_{t=1}^{m}\left(2^{i_{t}}-\right.$ $\left.2^{j_{t}}\right)$. For instance, take $n_{0}=\#\left\{D_{0}\right\}=42$ that is nearer to the number $2^{5}$ than $2^{6}$; we have

$$
n_{0}=2^{5}+2^{3}+2=\left(2^{6}-2^{5}\right)+\left(2^{4}-2^{3}\right)+\left(2^{2}-2\right) .
$$

Thus $i_{1}=6, j_{1}=5, i_{2}=4, j_{2}=3, i_{3}=2$, and $j_{3}=1$. And take $n_{0}=54$ which is closer to the number $2^{6}$ than $2^{5}$; one obtains $n_{0}=2^{6}-2^{4}+6=2^{6}-2^{4}+2^{3}-2$. Then $i_{1}=6, j_{1}=4, i_{2}=3$, and $j_{2}=1$.

Consider Case 2 of $D_{0}$. Denote

$$
\mathscr{H}(t)=a_{i_{t}}\left(\cdots\left(a_{i_{m-1}}\left(a_{i_{m}} H_{i_{m}} \cup D_{m-1}\right) \cdots\right) \cup D_{t}\right)
$$

for $1<t \leq m$. Clearly, $\mathscr{H}(t) \subset H_{j_{t-1}}$ and $\mathscr{H}(1)=D_{0}$ in Case 2. For two factors $d_{1} \in \mathscr{H}(t)$ and $d_{2} \in D_{t-1}$, one has $d_{1} d_{2} \in D_{t-1}$. Therefore,

$$
\begin{array}{r}
\#\left\{\left(d_{1}, d_{2}\right): d_{1} \in \mathscr{H}(t), d_{2} \in D_{t-1}, d_{1} d_{2}=\gamma\right\} \\
= \begin{cases}\sum_{l=t}^{m-1}\left(2^{i_{l}}-2^{j_{l}}\right)+2^{i_{m}}-1, & \gamma \in D_{t-1}, \\
0, & \gamma \notin D_{t-1} .\end{cases}
\end{array}
$$

Specifically, if $t=m$, then

$$
\begin{gathered}
\#\left\{\left(d_{1}, d_{2}\right): d_{1} \in a_{i_{m}} H_{i_{m}}, d_{2} \in D_{m-1}, d_{1} d_{2}=\gamma\right\} \\
= \begin{cases}2^{i_{m}}-1, & \gamma \in D_{m-1}, \\
0, & \gamma \notin D_{m-1} .\end{cases}
\end{gathered}
$$

For $m \geq 1$ and $\gamma \in H_{i_{m}}$, there are $2^{i_{m}-1}-1$ pairs of factors in $H_{i_{m}}$, which each interaction is aliased with $\gamma$. Then

$$
\begin{aligned}
B_{2}\left(a_{i_{m}} H_{i_{m}}, \gamma\right) & =B_{2}\left(H_{i_{m}}, \gamma\right) \\
& = \begin{cases}2^{i_{m}-1}-1, & \gamma \in H_{i_{m}}, \\
0, & \gamma \in H_{q} \backslash H_{i_{m}} .\end{cases}
\end{aligned}
$$

Based on the above results, we can obtain the value of $B_{2}\left(D_{0}, \gamma\right)$ for any $\gamma \in H_{q}$ in Case 2 .

Lemma 4. Let $D_{0}=a_{i_{1}}\left(a_{i_{2}}\left(\cdots\left(a_{i_{m-1}}\left(a_{i_{m}} H_{i_{m}} \cup D_{m-1}\right) \cdots\right) \cup\right.\right.$ $\left.\left.D_{2}\right) \cup D_{1}\right)$. Then

$$
\begin{aligned}
& B_{2}\left(D_{0}, \gamma\right) \\
& = \begin{cases}\frac{c(m)}{2}+2^{i_{m}-1}-1, & \gamma \in H_{i_{m}}, \\
c(m)-\frac{\left(c(t)+2^{i_{t}}\right)}{2}+2^{i_{m}}-1, & \gamma \in D_{t}, t=1, \ldots, m-1, \\
\frac{c(t)}{2}, & \gamma \in H_{j_{t-1}} \backslash H_{i_{t}}, t=2, \ldots, m, \\
0, & \gamma \in H_{q} \backslash H_{i_{1}},\end{cases}
\end{aligned}
$$

where $c(t)$ is defined in (14).
Proof. By (22), we obtain

$$
\begin{aligned}
B_{2}\left(D_{0}, \gamma\right) & \\
= & \sum_{l=1}^{m-1} B_{2}\left(D_{l}, \gamma\right)+B_{2}\left(a_{i_{m}} H_{i_{m}}, \gamma\right) \\
& +\#\left\{\left(d_{1}, d_{2}\right): d_{1} \in a_{i_{m}} H_{i_{m}}, d_{2} \in D_{m-1}, d_{1} d_{2}=\gamma\right\} \\
& +\sum_{l=2}^{m-1} \#\left\{\left(d_{1}, d_{2}\right): d_{1} \in \mathscr{H}(l), d_{2} \in D_{l-1}, d_{1} d_{2}=\gamma\right\} .
\end{aligned}
$$

For $\gamma \in H_{q} \backslash H_{i_{1}}$, we have $B_{2}\left(D_{0}, \gamma\right)=0$. By Lemma 2, (25), and (23), analyze the following cases.

(i) For $\gamma \in H_{i_{m}}$, we obtain

$$
\begin{aligned}
B_{2}\left(D_{0}, \gamma\right) & =\sum_{l=1}^{m-1} B_{2}\left(D_{l}, \gamma\right)+B_{2}\left(a_{i_{m}} H_{i_{m}}, \gamma\right) \\
& =\sum_{l=1}^{m-1}\left(2^{i_{l}-1}-2^{j_{l}-1}\right)+2^{i_{m}-1}-1 .
\end{aligned}
$$

(ii) For $\gamma \in D_{t}$ with $1 \leq t \leq m-1$, one has

$$
\begin{aligned}
B_{2}\left(D_{0}, \gamma\right) & \\
= & \sum_{l=1}^{t-1} B_{2}\left(D_{l}, \gamma\right)+B_{2}\left(D_{t}, \gamma\right) \\
& +\#\left\{\left(d_{1}, d_{2}\right): d_{1} \in \mathscr{H}(t+1), d_{2} \in D_{t}, d_{1} d_{2}=\gamma\right\} \\
= & \sum_{l=1}^{t-1}\left(2^{i_{l}-1}-2^{j_{l}-1}\right) \\
& +\sum_{l=t}^{m-1}\left(2^{i_{l}}-2^{j_{l}}\right)-2^{i_{t}-1}+2^{i_{m}}-1 \\
= & c(m)-\frac{\left(c(t)+2^{i_{t}}\right)}{2}+2^{i_{m}}-1 .
\end{aligned}
$$

(iii) For $\gamma \in H_{j_{t-1}} \backslash H_{i_{t}}$ with $2 \leq t \leq m, B_{2}\left(D_{0}, \gamma\right)=$ $\sum_{l=1}^{t-1}\left(2^{i_{l}-1}-2^{j_{l}-1}\right)$.

In Lemma 4 , the value of $B_{2}\left(D_{0}, \gamma\right)$ is relative to these pairs $\left\{i_{t}, j_{t}\right\}_{1<t<m}$ and $i_{m}$. For example, consider $n_{0}=\#\left\{D_{0}\right\}=$ 21. Since $n_{0}=\left(2^{5}-2^{4}\right)+\left(2^{3}-2^{2}\right)+2-1$, it yields $i_{1}=5$, $j_{1}=4, i_{2}=3, j_{2}=2$, and $i_{3}=1$. Taking $n_{0}=29$, we have $n_{0}=2^{5}-3=2^{5}-2^{2}+1$; thus $i_{1}=5, j_{1}=2$, and $i_{2}=1$.

Lemmas 3 and 4 , respectively, obtain the value of $B_{2}\left(D_{0}, \gamma\right)$ that $D_{0}$ consists of the last $n_{0}$ columns of $T_{r}(r \leq q)$ for two cases. These results play a key role in calculating ${ }_{1}^{\#} C_{2}$ and ${ }_{2}^{\#} C_{2}$ 's for all GMC $2^{n-m}$ designs with $5 N / 16+1 \leq n \leq$ $N-1$. Next sections will, respectively, discuss two-level GMC designs with the factor number $n$ satisfying (i) $5 N / 16+1 \leq$ $n<N / 2$ or (ii) $N / 2 \leq n \leq N-1$. 


\section{GMC $2^{n-m}$ Designs with $5 N / 16+1 \leq n<N / 2$}

Li et al. [10] showed all GMC $2^{n-m}$ designs with $5 N / 16+1 \leq$ $n<N / 2$, constructed by the last $n$ columns of $T_{q}$. In Section 2 , $D_{0}$ is constructed by Case 1 or Case 2 , which is the last $n_{0}$ columns of $T_{r}(r \leq q)$ for $n_{0}=\#\left\{D_{0}\right\}$. Therefore, for any GMC $2^{n-m}$ design $D$ with $5 N / 16+1 \leq n<N / 2$, its construction is similar to that of $D_{0}$. In (3), take $j_{0}=r=q$.

Theorem 5. Consider GMC $2^{n-m}$ design

$$
D=a_{i_{1}}\left(a_{i_{2}}\left(\cdots\left(a_{i_{m-1}}\left(a_{i_{m}} D_{m} \cup D_{m-1}\right) \cdots\right) \cup D_{2}\right) \cup D_{1}\right)
$$

with $5 N / 16+1 \leq n<N / 2$. Then

(a)

$$
{ }_{1}^{\#} C_{2}^{(k)}(D)= \begin{cases}n, & k=0 \\ 0, & \text { otherwise }\end{cases}
$$

(b)

$$
\begin{aligned}
& { }_{2}^{\#} C_{2}^{(k)}(D) \\
& = \begin{cases}\frac{n\left(2^{j_{m}}-1\right)}{2}, & k=\frac{n}{2}-1, \\
\left(n-\frac{\left(c(t)+2^{i_{t}}\right)}{2}\right)\left(2^{i_{t}}-2^{j_{t}}\right), & k=n-\frac{\left(c(t)+2^{i_{t}}\right)}{2}-1, \\
\frac{c(t)\left(2^{j_{t-1}}-2^{i_{t}}\right)}{2}, & t=1, \ldots, m, \\
0, & k=\frac{c(t)}{2}-1, t=2, \ldots, m,\end{cases}
\end{aligned}
$$

where $c(t)$ is defined in (14).

Proof. Evidently, $n=\sum_{l=1}^{m}\left(2^{i_{l}}-2^{j_{l}}\right)$; we have $c(m+1)=n$. By Lemma 3,

$$
\begin{aligned}
& B_{2}(D, \gamma) \\
& = \begin{cases}\frac{n}{2}, & \gamma \in H_{i_{m}}, \\
n-\frac{\left(c(t)+2^{i_{t}}\right)}{2}, & \gamma \in D_{t}, t=1, \ldots, m, \\
\frac{c(t)}{2}, & \gamma \in H_{j_{t-1}} \backslash H_{i_{t}}, t=2, \ldots, m, \\
0, & \gamma \in H_{q} \backslash H_{i_{1}} .\end{cases}
\end{aligned}
$$

(a) Since $D \subset H_{q} \backslash H_{i_{1}}$, hence by (33) and (5)

$$
{ }_{1}^{\#} C_{2}^{(0)}(D)=\#\left\{\gamma: \gamma \in D, B_{2}(D, \gamma)=0\right\}=\#\{D\}=n .
$$

Otherwise, ${ }_{1}^{\#} C_{2}^{(k)}(D)=0$ for $k \neq 0$.

(b) Following (33) and (6), we obtain

$$
\begin{aligned}
{ }_{2}^{\#} C_{2}^{(k)}(D)= & (k+1) \\
& \times\left[\#\left\{\gamma: \gamma \in H_{j_{m}}, B_{2}(D, \gamma)=k+1\right\}\right.
\end{aligned}
$$

$$
\begin{aligned}
& +\sum_{t=1}^{m} \#\left\{\gamma: \gamma \in D_{t}, B_{2}(D, \gamma)=k+1\right\} \\
& +\sum_{t=2}^{m} \#\left\{\gamma: \gamma \in H_{j_{t-1}} \backslash H_{i_{t}}, B_{2}(D, \gamma)=k+1\right\} \\
& \left.+\#\left\{\gamma: \gamma \in H_{q} \backslash H_{i_{1}}, B_{2}(D, \gamma)=k+1\right\}\right] .
\end{aligned}
$$

If $k=n / 2-1$, then

$$
{ }_{2}^{\#} C_{2}^{(k)}(D)=\frac{n \#\left\{H_{j_{m}}\right\}}{2}=\frac{n\left(2^{j_{m}}-1\right)}{2} .
$$

For $k=n-\left(c(t)+2^{i_{t}}\right) / 2-1$ with $1 \leq t \leq m$, one has

$$
\begin{aligned}
{ }_{2}^{\#} C_{2}^{(k)}(D) & =\left(n-\frac{\left(c(t)+2^{i_{t}}\right)}{2}\right) \#\left\{D_{t}\right\} \\
& =\left(n-\frac{\left(c(t)+2^{i_{t}}\right)}{2}\right)\left(2^{i_{t}}-2^{j_{t}}\right) .
\end{aligned}
$$

And if $k=c(t) / 2-1$ with $1<t \leq m$, then

$$
{ }_{2}^{\#} C_{2}^{(k)}(D)=\frac{c(t) \#\left\{H_{j_{t-1}} \backslash H_{i_{t}}\right\}}{2}=\frac{c(t)\left(2^{j_{t-1}}-2^{i_{t}}\right)}{2} .
$$

Otherwise, ${ }_{2}^{\#} C_{2}^{(k)}(D)=0$.

For GMC $2^{n-m}$ design with $5 N / 16+1 \leq n<N / 2$, Theorem 5 reveals that the value of ${ }_{1}^{\#} C_{2}$ only depends on the factor number $n$. However, the value of ${ }_{2}^{\#} C_{2}$ is related to the numbers $\left\{i_{t}, j_{t}\right\}_{1 \leq t \leq m}$ besides $n$. We illustrate them via a simple example.

Example 6. Take $q=5$ and $n=10$; consider GMC $2^{10-5}$ design $D$. Since $n=2^{3}+2=\left(2^{4}-2^{3}\right)+\left(2^{2}-2\right)$, clearly, we have $i_{1}=4, j_{1}=3, i_{2}=2$, and $j_{2}=1$. Hence, $2^{i_{1}}-2^{j_{1}}=8$, $2^{i_{2}}-2^{j_{2}}=2,2^{j_{1}}-2^{i_{2}}=4$, and $c(1)=0, c(2)=8$. By Theorem 5 , we get

$$
\begin{aligned}
& { }_{1}^{\#} C_{2}^{(k)}(D)= \begin{cases}10, & k=0, \\
0, & \text { otherwise, }\end{cases} \\
& { }_{2}^{\#} C_{2}^{(k)}(D)= \begin{cases}16, & k=1, \\
24, & k=3, \\
5, & k=4, \\
0, & \text { otherwise. }\end{cases}
\end{aligned}
$$

Theorem 5 applies to the case that the factor number $n$ of GMC design is even. If $n$ is odd, similar to the proof of Theorem 5, by Lemma 4, one can get the result below.

Theorem 7. Consider GMC $2^{n-m}$ design

$$
D=a_{i_{1}}\left(a_{i_{2}}\left(\cdots\left(a_{i_{m-1}}\left(a_{i_{m}} H_{i_{m}} \cup D_{m-1}\right) \cdots\right) \cup D_{2}\right) \cup D_{1}\right)
$$

with $5 N / 16+1 \leq n<N / 2$. Then 
(a)

$$
{ }_{1}^{\#} C_{2}^{(k)}(D)= \begin{cases}n, & k=0 \\ 0, & \text { otherwise }\end{cases}
$$

(b)

$$
\begin{aligned}
& { }_{2}^{\#} C_{2}^{(k)}(D) \\
& = \begin{cases}\frac{(n-1)\left(2^{i_{m}}-1\right)}{2}, & k=\frac{(n-1)}{2}-1, \\
\left(n-\frac{\left(c(t)+2^{i_{t}}\right)}{2}\right)\left(2^{i_{t}}-2^{j_{t}}\right), & k=n-\frac{\left(c(t)+2^{i_{t}}\right)}{2}-1, \\
\frac{c(t)\left(2^{j_{t-1}}-2^{i_{t}}\right)}{2}, & t=1, \ldots, m-1, \\
0, & k=\frac{c(t)}{2}-1, t=2, \ldots, m, \\
\text { otherwise },\end{cases}
\end{aligned}
$$

where $c(t)$ is defined in (14).

Proof. Note that $n=\sum_{l=1}^{m-1}\left(2^{i_{l}}-2^{j_{l}}\right)+2^{i_{m}}-1=c(m)+2^{i_{m}}-$ 1.

Example 8. Let $q=5$ and $n=11$; consider GMC $2^{11-6}$ design $D$. Here $n=2^{4}-2^{3}+2^{2}-1$; we have $i_{1}=4, j_{1}=3$, and $i_{2}=2$. Thus, $c(2)=2^{i_{1}}-2^{j_{1}}=8$ and $2^{j_{1}}-2^{i_{2}}=4$. Following Theorem 7 , it is directly obtained by

$$
\begin{aligned}
& { }_{1}^{\#} C_{2}^{(k)}(D)= \begin{cases}11, & k=0, \\
0, & \text { otherwise }\end{cases} \\
& { }_{2}^{\#} C_{2}^{(k)}(D)= \begin{cases}24, & k=2, \\
16, & k=3, \\
15, & k=4, \\
0, & \text { otherwise. }\end{cases}
\end{aligned}
$$

\section{GMC $2^{n-m}$ Designs with $N / 2 \leq n \leq N-1$}

In Section 2, we know that any GMC $2^{n-m}$ design with $N / 2 \leq$ $n \leq N-1$ is constructed by $D_{0} \cup S_{q r}$, where $D_{0}$ is the last $n-\left(N-2^{r}\right)$ columns of $T_{r}(r<q)$. Lemmas 3 and 4 have shown the confounding information of $D_{0}$. Next we will study a special design $S_{q r}=H_{q} \backslash H_{r}(r<q)$, which consists of the last $N-2^{r}$ columns of $H_{q}$. Since $r<q$, the factor number of the design $S_{q r}$ satisfies $N-2^{r} \geq N / 2$. Hence, the design $S_{q r}$ has GMC. By Lemma 2, we directly give the value of $B_{2}\left(S_{q r}, \gamma\right)$ as follows:

$$
B_{2}\left(S_{q r}, \gamma\right)= \begin{cases}\frac{N}{2}-2^{r}, & \gamma \in H_{q} \backslash H_{r}, \\ \frac{N}{2}-2^{r-1}, & \gamma \in H_{r} .\end{cases}
$$

Next we discuss the values of ${ }_{1}^{\#} C_{2}$ and ${ }_{2}^{\#} C_{2}$ for GMC design $S_{q r}$ with $r<q$.
Theorem 9. Consider any GMC design $S_{q r}=H_{q} \backslash H_{r}$ for $r<q$. Then

(a)

$$
{ }_{1}^{\#} C_{2}^{(k)}\left(S_{q r}\right)= \begin{cases}N-2^{r}, & k=\frac{N}{2}-2^{r} \\ 0, & \text { otherwise }\end{cases}
$$

(b)

$$
{ }_{2}^{\#} C_{2}^{(k)}\left(S_{q r}\right)= \begin{cases}\left(\frac{N}{2}-2^{r}\right)\left(N-2^{r}\right), & k=\frac{N}{2}-2^{r}-1, \\ \left(\frac{N}{2}-2^{r-1}\right)\left(2^{r}-1\right), & k=\frac{N}{2}-2^{r-1}-1, \\ 0, & \text { otherwise. }\end{cases}
$$

Proof. (a) If $k=N / 2-2^{r}$, by (44), then

$$
\begin{aligned}
{ }_{1}^{\#} C_{2}^{(k)}\left(S_{q r}\right) & =\#\left\{\gamma: \gamma \in S_{q r}, B_{2}\left(S_{q r}, \gamma\right)=k\right\} \\
& =\#\left\{S_{q r}\right\}=N-2^{r} .
\end{aligned}
$$

Otherwise, ${ }_{1}^{\#} C_{2}^{(k)}\left(S_{q r}\right)=0$.

(b) For $k \geq 0$, note that

$$
\begin{aligned}
{ }_{2}^{\#} C_{2}^{(k)}\left(S_{q r}\right)= & (k+1) \\
& \times\left[\#\left\{\gamma: \gamma \in S_{q r}, B_{2}\left(S_{q r}, \gamma\right)=k+1\right\}\right. \\
& \left.+\#\left\{\gamma: \gamma \in H_{r}, B_{2}\left(S_{q r}, \gamma\right)=k+1\right\}\right] .
\end{aligned}
$$

If $k=N / 2-2^{r}-1$, thus by (44)

$$
{ }_{2}^{\#} C_{2}^{(k)}\left(S_{q r}\right)=\left(\frac{N}{2}-2^{r}\right) \#\left\{S_{q r}\right\}=\left(\frac{N}{2}-2^{r}\right)\left(N-2^{r}\right) .
$$

Similarly, for $k=N / 2-2^{r-1}-1$, we have

$$
{ }_{2}^{\#} C_{2}^{(k)}\left(S_{q r}\right)=\left(\frac{N}{2}-2^{r-1}\right) \#\left\{H_{r}\right\}=\left(\frac{N}{2}-2^{r-1}\right)\left(2^{r}-1\right) .
$$

For GMC design $S_{q r}(r<q)$, the values of ${ }_{1}^{\#} C_{2}$ and ${ }_{2}^{\#} C_{2}$ only rely on two numbers $N$ and $r$. In particular, if $r=q-1$, then $S_{q(q-1)}=T_{q}$. By Theorem 9, one has

$$
{ }_{1}^{\#} C_{2}^{(k)}\left(T_{q}\right)= \begin{cases}\frac{N}{2}, & k=0, \\ 0, & k \neq 0,\end{cases}
$$




$$
{ }_{2}^{\#} C_{2}^{(k)}\left(T_{q}\right)= \begin{cases}\frac{N}{4(N / 2-1)}, & k=\frac{N}{4}-1, \\ 0, & k \neq \frac{N}{4}-1 .\end{cases}
$$

The next example is used to illustrate this above result.

Example 10. Consider GMC $2^{16-11}$ design $S_{54}$. Since $r=4$ and $N=32$, one directly gets

$$
\begin{aligned}
& { }_{1}^{\#} C_{2}^{(k)}(D)= \begin{cases}16, & k=0, \\
0, & k \neq 0,\end{cases} \\
& { }_{2}^{\#} C_{2}^{(k)}(D)= \begin{cases}120, & k=7, \\
0, & k \neq 7 .\end{cases}
\end{aligned}
$$

On the other hand, every GMC $2^{n-m}$ design $D$ with $n \geq$ $N / 2$ can be constructed by the form $\left(D \backslash S_{q r}\right) \cup S_{q r}$, where $D \backslash S_{q r}$ consists of the last $n-\left(N-2^{r}\right)$ columns of $T_{r}$. Then, $D_{0}=D \backslash S_{q r}$. Based on Lemma 3 of Li et al. [10], we obtain the relationship of $D$ and $D_{0}$ as follows:

$$
B_{2}(D, \gamma)= \begin{cases}n-\frac{N}{2}, & \gamma \in H_{q} \backslash H_{r} \\ B_{2}\left(D_{0}, \gamma\right)+\frac{N}{2}-2^{r-1}, & \gamma \in H_{r}\end{cases}
$$

Therefore, we can get the following result.

Theorem 11. Consider GMC $2^{n-m}$ design $D=D_{0} \cup S_{q r}$ with $r<$ $q$, where $D_{0}=a_{i_{1}}\left(a_{i_{2}}\left(\cdots\left(a_{i_{m-1}}\left(a_{i_{m}} D_{m} \cup D_{m-1}\right) \cdots\right) \cup D_{2}\right) \cup D_{1}\right)$. Then

(a)

$$
B_{2}(D, \gamma)= \begin{cases}\frac{n}{2}, & \gamma \in H_{j_{m}}, \\ n-\frac{\left(a(t)+2^{i_{t}}\right)}{2}, & \gamma \in D_{t}, t=1, \ldots, m, \\ \frac{a(t)}{2}, & \gamma \in H_{j_{t-1}} \backslash H_{i_{t}}, t=2, \ldots, m, \\ n-\frac{N}{2}, & \gamma \in H_{r} \backslash H_{i_{1}}, \\ & \gamma \in H_{q} \backslash H_{r},\end{cases}
$$

(b)

$$
{ }_{1}^{\#} C_{2}^{(k)}(D)= \begin{cases}N-2^{r}, & k=n-\frac{N}{2}, \\ n-N+2^{r}, & k=\frac{N}{2}-2^{r-1}, \\ 0, & \text { otherwise, }\end{cases}
$$

(c)

$$
{ }_{2}^{\#} C_{2}^{(k)}(D)
$$

$$
= \begin{cases}\frac{n\left(2^{j_{m}}-1\right)}{2}, & k=\frac{n}{2}-1, \\ \left(n-\frac{\left(a(t)+2^{i_{t}}\right)}{2}\right)\left(2^{i_{t}}-2^{j_{t}}\right), & k=n-\frac{\left(a(t)+2^{i_{t}}\right)}{2}-1, \\ \frac{a(t)\left(2^{j_{t-1}}-2^{i_{t}}\right)}{2}, & k=1, \ldots, m, \\ \left(\frac{N}{2}-2^{r-1}\right)\left(2^{r}-2^{i_{1}}\right), & t=2, \ldots, m, \\ \left(n-\frac{N}{2}\right)\left(N-2^{r}\right), & k=\frac{N}{2}-2^{r-1}-1, \\ 0, & k=n-\frac{N}{2}-1,\end{cases}
$$

where $a(t)=N-2^{r}+c(t)$ and $c(t)$ is defined in (14).

Proof. (a) By (53) and Lemma 3, note that

$$
c(m+1)=\#\left\{D_{0}\right\}=\sum_{l=1}^{m}\left(2^{i_{l}}-2^{j_{l}}\right)=n-N+2^{r}
$$

yields (a).

(b) For $\gamma \in S_{q r}$, by (a), $B_{2}(D, \gamma)=n-N / 2$. If $k=n-N / 2$, then

$$
{ }_{1}^{\#} C_{2}^{(k)}(D)=\#\left\{S_{q r}\right\}=N-2^{r}
$$

Since $D_{0} \subset H_{r} \backslash H_{i_{1}}$, for $k=N / 2-2^{r-1}$, we have

$$
\begin{aligned}
{ }_{1}^{\#} C_{2}^{(k)}(D) & =\#\left\{D_{0}\right\} \\
& =\#\left\{\bigcup_{t=1}^{m} D_{t}\right\}=\sum_{t=1}^{m}\left(2^{i_{t}}-2^{j_{t}}\right)=n-N+2^{r} .
\end{aligned}
$$

(c) Since

$$
\begin{aligned}
{ }_{2}^{\#} C_{2}^{(k)}(D)=( & +1) \\
\times & {\left[\left\{\gamma: \gamma \in H_{j_{m}}, B_{2}(D, \gamma)=k+1\right\}\right.} \\
& +\sum_{t=1}^{m} \#\left\{\gamma: \gamma \in D_{l}, B_{2}(D, \gamma)=k+1\right\} \\
& +\sum_{t=2}^{m} \#\left\{\gamma: \gamma \in H_{j_{t-1}} \backslash H_{i_{k}}, B_{2}(D, \gamma)=k+1\right\} \\
& +\#\left\{\gamma: \gamma \in H_{r} \backslash H_{i_{1}}, B_{2}(D, \gamma)=k+1\right\} \\
& \left.+\#\left\{\gamma: \gamma \in H_{q} \backslash H_{r}, B_{2}(D, \gamma)=k+1\right\}\right],
\end{aligned}
$$

by (a), the result follows. 
When the factor number $n$ of a GMC design satisfying $N / 2 \leq n \leq N-1$ is even, by Theorem 11, we obtain values of the corresponding ${ }_{1}^{\#} C_{2}$ and ${ }_{1}^{\#} C_{2}$. The next example illustrates this point.

Example 12. Let $q=8, r=7$; consider GMC $2^{154-146}$ design $D=D_{0} \cup S_{87}$. Since $n_{0}=\#\left\{D_{0}\right\}=26$ and

$$
n_{0}=2^{5}-6=\left(2^{5}-2^{3}\right)+\left(2^{2}-2\right),
$$

we have $i_{1}=5, j_{1}=3, i_{2}=2$, and $j_{2}=1$. Thus, $2^{i_{1}}-2^{j_{1}}=$ $24,2^{i_{2}}-2^{j_{2}}=2$ and $a(1)=N-2^{r}+c(1)=128, a(2)=$ $N-2^{r}+c(2)=152$. By (b) and (c) of Theorem 11, one obtains

$$
\begin{aligned}
& { }_{1}^{\#} C_{2}^{(k)}(D)= \begin{cases}128, & k=26, \\
26, & k=64, \\
0, & \text { otherwise, }\end{cases} \\
& { }_{2}^{\#} C_{2}^{(k)}(D)= \begin{cases}3328, & k=25, \\
6144, & k=63, \\
1776, & k=73, \\
456, & k=75, \\
77 & k=76, \\
0, & \text { otherwise. }\end{cases}
\end{aligned}
$$

Theorem 13. Consider GMC $2^{n-m}$ design $D=D_{0} \cup S_{q r}$ with $r<q$, where $D_{0}=a_{i_{1}}\left(a_{i_{2}}\left(\cdots\left(a_{i_{m-1}}\left(a_{i_{m}} H_{i_{m}} \cup D_{m-1}\right) \cdots\right) \cup D_{2}\right) \cup\right.$ $\left.D_{1}\right)$. Then

(a)

$$
\begin{aligned}
& B_{2}(D, \gamma) \\
& =\left\{\begin{array}{ll}
\frac{(n-1)}{2}, & \gamma \in H_{i_{m}}, \\
\frac{a(t)}{2}, & \gamma \in D_{t}, t=1, \ldots, m-1, \\
\frac{N}{2}-2^{r-1}, & \gamma \in H_{j_{t-1}} \backslash H_{i_{t}}, t=2, \ldots, m, \\
n-\frac{N}{2}, & \gamma \in H_{r} \backslash H_{i_{1}}, \\
&
\end{array}, H_{r},\right.
\end{aligned}
$$

(b)

$$
{ }_{1}^{\#} C_{2}^{(k)}(D)= \begin{cases}N-2^{r}, & k=n-\frac{N}{2}, \\ n-N+2^{r}, & k=\frac{N}{2}-2^{r-1}, \\ 0, & \text { otherwise, }\end{cases}
$$

(c)

$$
\begin{aligned}
& { }_{2}^{\#} C_{2}^{(k)}(D) \\
& = \begin{cases}\frac{(n-1)\left(2^{i_{m}}-1\right)}{2}, & k=\frac{(n-1)}{2}-1, \\
\left(n-\frac{\left(a(t)+2^{i_{t}}\right)}{2}\right)\left(2^{i_{t}}-2^{j_{t}}\right), & k=n-\frac{\left(a(t)+2^{i_{t}}\right)}{2}-1, \\
\frac{a(t)\left(2^{j_{t-1}}-2^{i_{t}}\right)}{2}, & t=1, \ldots, m-1, \\
\left(\frac{N}{2}-2^{r-1}\right)\left(2^{r}-2^{i_{1}}\right), & k=\frac{a(t)}{2}-1, \\
\left(n-\frac{N}{2}\right)\left(N-2^{r}\right), & t=2, \ldots, m, \\
0, & k=\frac{N}{2}-2^{r-1}-1, \\
& k=n-\frac{N}{2}-1, \\
\text { otherwise, }\end{cases}
\end{aligned}
$$

where $a(t)=N-2^{r}+c(t)$ and $c(t)$ is defined in (14).

Proof. Only prove (a). Since

$$
\#\left\{D_{0}\right\}=\sum_{l=1}^{m-1}\left(2^{i_{l}}-2^{j_{l}}\right)+2^{i_{m}}-1=n-N+2^{r} \text {, }
$$

one has $c(m)=n-\left(N-2^{r}\right)-\left(2^{i_{m}}-1\right)$. By (53) and Lemma 4, yields (a).

The proof of (b) and (c) is similar to those of Theorem 11. The following example serves to show its application.

Example 14. Let $q=8, r=7$, and $N=256$ and consider GMC $2^{135-127}$ design $D=D_{0} \cup S_{87}$. Since $\#\left\{D_{0}\right\}=2^{3}-1$, we have $i_{1}=3$. By (b) and (c) of Theorem 13, one gets

$$
\begin{aligned}
& { }_{1}^{\#} C_{2}^{(k)}(D)= \begin{cases}128, & k=7, \\
7, & k=64, \\
0, & \text { otherwise, }\end{cases} \\
& { }_{2}^{\#} C_{2}^{(k)}(D)= \begin{cases}896, & k=6, \\
7680, & k=63, \\
469, & k=66, \\
0, & \text { otherwise. }\end{cases}
\end{aligned}
$$

\section{Concluding Remark}

Based on construction of GMC $2^{n-m}$ designs with $5 N / 16+$ $1 \leq n \leq N-1$, we obtain the mathematical formulation to calculate the values of ${ }_{1}^{\#} C_{2}$ and ${ }_{2}^{\#} C_{2}$ in the AENP. These results are very useful to analyze the confounding information among lower-order factors of two-level GMC designs. For GMC $2^{n-m}$ designs satisfying $n \notin[5 N / 16+1, N-1]$, some further studies in this direction are in progress. 


\section{Conflict of Interests}

The authors declare that there is no conflict of interests regarding the publication of this paper.

\section{Acknowledgments}

This work was partially supported by the National Natural Science Foundation of China Grants 11171165, 10871104, and 11101074; Scientific Research Program of the Higher Education Institution of XinJiang Grant XJEDU2012S01; and Research Fund for the Doctoral Program of XinJiang University Grant BS130106. The authors are very grateful to the editor, the associate editor, and the referees for their valuable comments which have led to a substantial improvement in the paper.

\section{References}

[1] R. C. Zhang, P. Li, S. L. Zhao, and M. Y. Ai, "A general minimum lower-order confounding criterion for two-level regular designs," Statistica Sinica, vol. 18, no. 4, pp. 1689-1705, 2008.

[2] G. E. P. Box and J. S. Hunter, "The $2^{k-p}$ fractional factorial designs part I and II," Technometrics, vol. 3, pp. 311-458, 1961.

[3] A. Fries and W. G. Hunter, "Minimum aberration $2^{k-p}$ designs," Technometrics, vol. 22, no. 4, pp. 601-608, 1980.

[4] C. F. J. Wu and Y. Chen, "A graph-aided method for planning two-level experiments when certain interactions are important," Technometrics, vol. 34, pp. 162-175, 1992.

[5] D. X. Sun, Estimation capacity and related topics in experimental designs [Ph. D. thesis], University of Waterloo, Waterloo, Canada, 1993.

[6] R. C. Zhang and Y. Cheng, "General minimum lower order confounding designs: an overview and a construction theory," Journal of Statistical Planning and Inference, vol. 140, no. 7, pp. 1719-1730, 2010.

[7] J. W. Hu and R. C. Zhang, "Some results on two-level regular designs with general minimum lower-order confounding," Journal of Statistical Planning and Inference, vol. 141, no. 5, pp. 1774-1782, 2011.

[8] J. Chen and M. Q. Liu, "Some theory for constructing general minimum lower order confounding designs," Statistica Sinica, vol. 21, no. 4, pp. 1541-1555, 2011.

[9] Y. Cheng and R. C. Zhang, "On construction of general minimum lower order confounding $2^{n-m}$ designs with $N / 4+1 \leq$ $n \leq 9 \mathrm{~N} / 32$," Journal of Statistical Planning and Inference, vol. 140, pp. 2384-2394, 2010.

[10] P. F. Li, S. L. Zhao, and R. C. Zhang, "A theory on constructing $2^{n-m}$ designs with general minimum lower order confounding," Statistica Sinica, vol. 21, no. 4, pp. 1571-1589, 2011.

[11] R. Mukerjee and C. F. J. Wu, A Modern Theory of Factorial Designs, Springer, New York, NY, USA, 2006. 


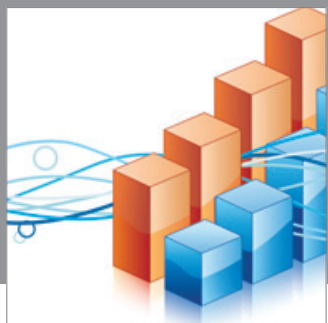

Advances in

Operations Research

mansans

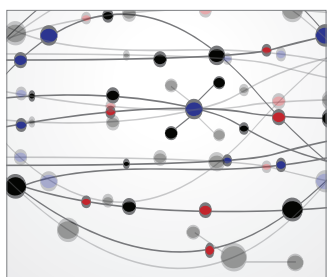

The Scientific World Journal
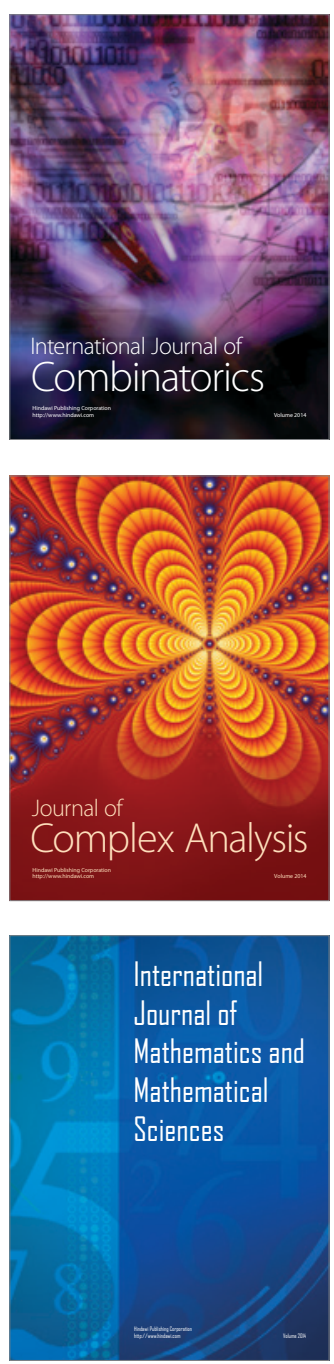
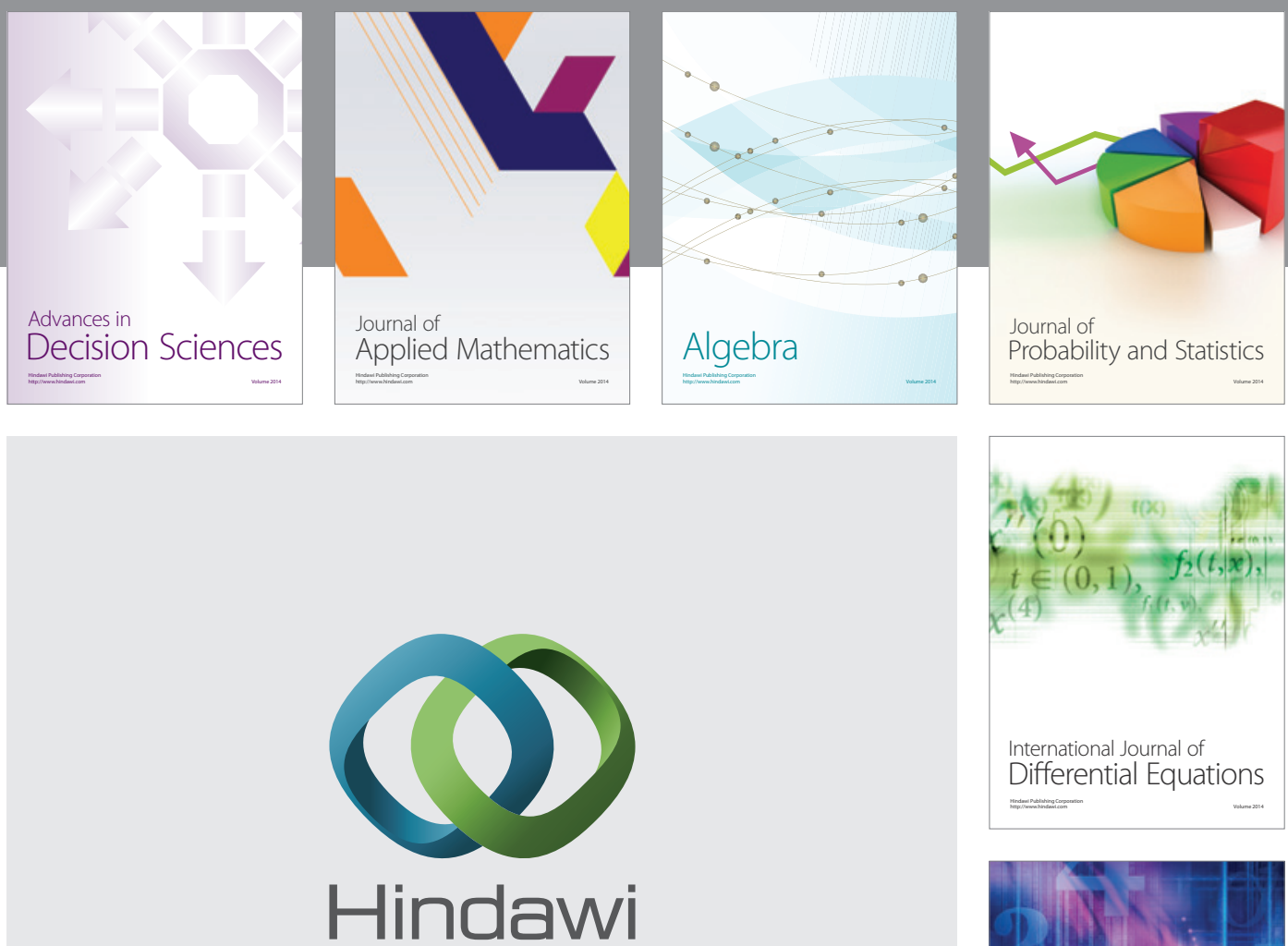

Submit your manuscripts at http://www.hindawi.com
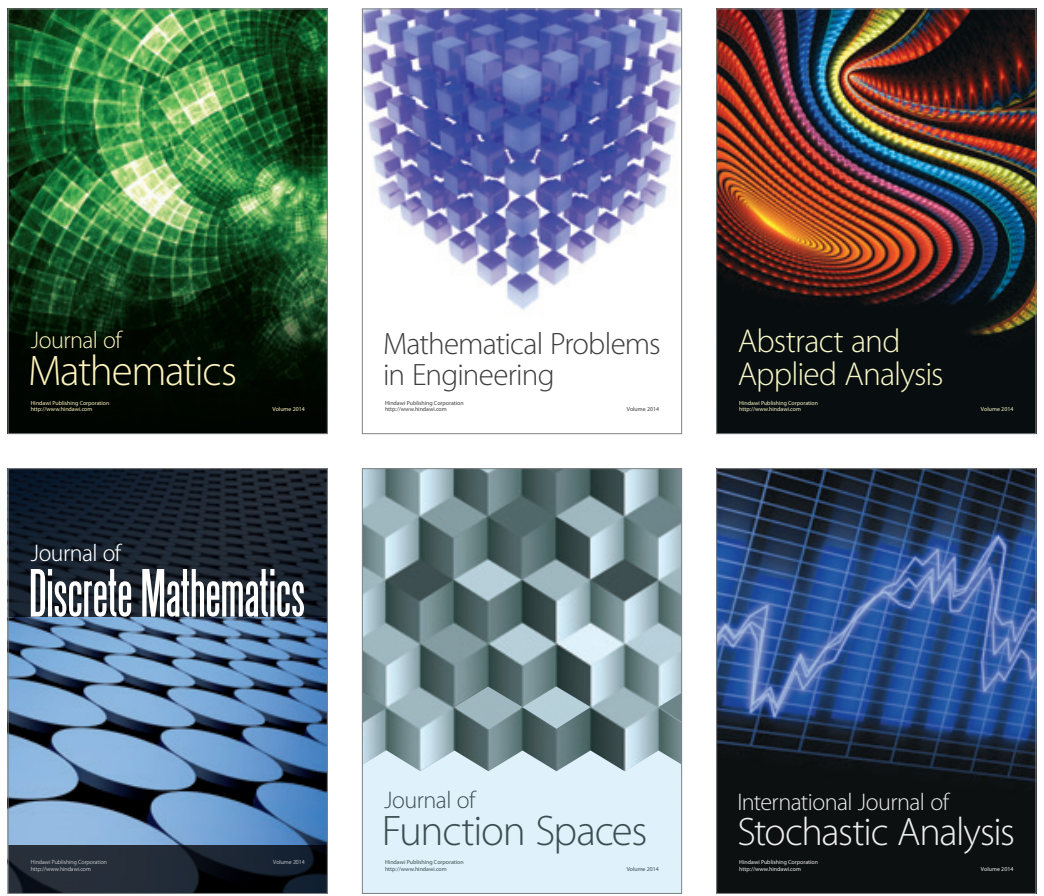

Journal of

Function Spaces

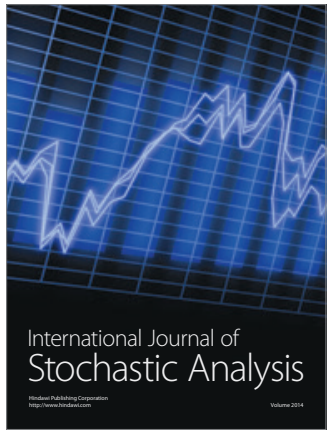

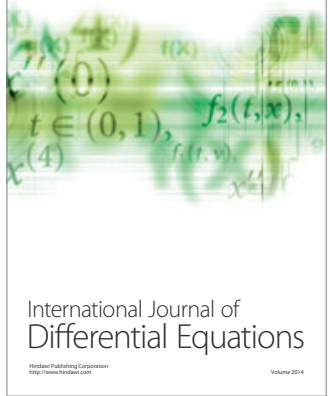
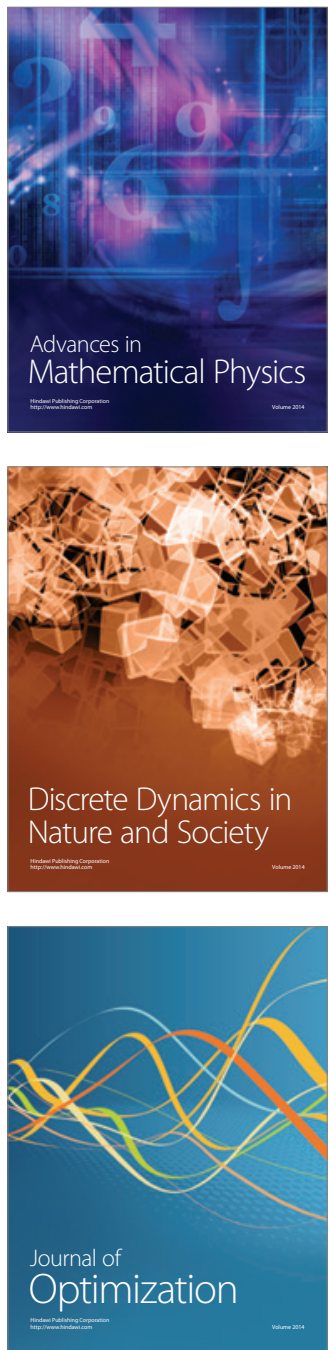